\title{
On Achievable QoS Bounds for Mobile IP through Rayleigh Channel in VANET
}

\author{
Richa Sharma \\ CSE Department, Guru Nanak Dev University Regional Campus, Jalandhar, INDIA \\ E-mail: Rixs11111991@g mail.com \\ Jyoteesh Malhotra \\ CSE Department, Guru Nanak Dev University Regional Campus, Jalandhar, INDIA \\ E-mail: Jyoteesh@gmail.com
}

\begin{abstract}
Vehicular Ad Hoc network is a versatile mobile wireless Ad-Hoc network targeted to support traffic monitoring, vehicular safety and many more applications. For the robust and reliable services in the VANET there is need to investigate the performance under frequent handovers in Mobile IP to prevent packet loss. Mobile IP is an interface that helps to track the mobile nodes and deliver messages even if vehicles are out of the coverage area of home node. In order to find the achievable performance bounds in terms of throughput, packet drop, collision rate and packet broadcast rate, extensive simulations have been done. A realistic city scenario has been proposed here by using the Rayleigh channel simulator, mobile IP enabled IEEE 802.11p OBUs and RSUs. The transmission powers of RSUs and threshold power levels have been varied to obtain the optimum performance through realistic conditions. Simulations are performed using NCTUns6.0 (National Chiao Tung University Network Simulator) in mobile IP interface.
\end{abstract}

Index Terms-VANET, Mobile IP, NCTUns6.0, 802.11p, Rayleigh Fading, Transmission power.

\section{INTRODUCTION}

VANET (Vehicular Ad-Hoc Network) are an instantiation of MANET (Mobile Ad-Hoc Network). The key distinction between VANET and MANET are swift changing topology, high mobility pattern and mobility prediction capabilities. VANET provides wireless communication among vehicles $(\mathrm{V} 2 \mathrm{~V})$ and vehicle to roadside units [4]. Internet connectivity among vehicles is main requirement of future communication networks. VANET is an infrastructure network where as internet is infrastructure with wired and fixed gateways which makes communication unreliable. For connecting heterogeneous networks some considerations should be taken into account. Firstly to address packets for transfer between infrastructure network and mobile nodes packet addressing should be done. To support this technique Mobile IP is a current standard which supports IP mobility of mobile nodes in the wireless network with infrastructure even in foreign network without breaking connection. Mobile IP enables the mobile node to change its access point without losing connection and access internet without disruption [2]. Mobile nodes should be in the coverage range of mobile IP base station. Secondly quality of service (QoS) support in VANET to send and receive data packets should be performed in particular time variants. Quality of service parameters in VANET need to be evaluated to check the service requirements that need to be met by the network while transporting a packet stream from source to destination [6][7].

VANET prototyping in real platform is a challenging task. A number of evaluations have been done in last few years, yet very few have considered mobile IP interface for systematic performance evaluation. Multi Protocol Label Switching (MPLS) has been combined with mobile IP in [4] to improve QoS in terms of throughput, packet loss and delay for traffic safety in urban areas. A lot of road side units (RSU) are used to simulate the environment using SUMO. The proposed framework has improved packet loss and throughput but no positive change in case of delay. Detection of failure and stationary node in mobile IP framework is performed in [5]. The main accent of this paper is on timely broadcasting of safety critical alerts to nearby vehicles and concluded that failure nodes has high number of collision packets with respect to moving nodes. Performance investigations have been reported considering mobile IP framework in the past [1] [6] but the work was limited to ideal channel conditions. In order to investigate the performance of VANET with mobile IP enabled nodes through realistic channel conditions work has been done here. In doing so Rayleigh channel simulator has been incorporated and power levels of OBU have been varied. So this work intends to extends the work related to mobile IP by evaluating the QoS parameters through realistic fading channel for creating realistic situations unlike the previous work that was limited to ideal channel moreover the power levels of RSU and threshold levels of OBU has been marked to compute the QoS has also been marked to compute the QoS parameters.

The rest of the paper is structured as follows: In section II throws light on the problem statement which arises in the VANET framework. Section III presents brief 
overview of the simulation methodology used. Section IV shows the results obtained in with variable power and threshold in Rayleigh fading environment under results and discussion. At last section $\mathrm{V}$ concludes the paper.

\section{PRoBlem St ATEMENT}

Vehicular networks are envisioned for the continuous access to IP based applications and drive through internet. All these services are supported by road side units (RSU) that connects the VANET to foreign IP networks. Yet VANET suffers from asymmetric links due to frequent handovers caused by obstacles, mobility and dissimilar transmission powers, this leads to disruptions in maintaining bidirectional connections. This problem is somehow resolved by using mobile IP. Mobile IP is a current standard which supports IP mobility of mobile nodes in the wireless network with infrastructure even in foreign network without breaking connection. It enables the mobile node to change its access point without losing connection and access internet without disruption. Dynamically addressing should be done when vehicles moves from coverage area of its base station or access point [2]. When packets fail to reach to destination due to static addressing it has bad effect on QoS parameters, as packet drop increases due to failure to reach to destination under time variants also overall throughput of network also decreases. In this paper dynamic path movement is selected to move vehicles to any direction at any time which helps to decrease variants in throughput rate.

\section{Simulation MethodologY AND ENVIRONMENT}

In order to compute the performance bounds through realistic channel conditions in VANET with mobile IP enabled nodes the following simulation scenario and methodology has been presented in this section. Simulator is a hardware or software application that provides an integrated, reliable and virtual environment to evaluate various QoS performance parameters in a network. NCTUns 6.0 provides simulation platform in this research work.

\section{A. Performance parameters}

The following QoS parameters are used to evaluate the performance of VANET in mobile IP interface by varying power and threshold.

- Packet drop ratio basically defines the total number of packets failed to reach to its destination. Many factors like congestion, frequent fading, queue overloading and varying threshold can be reasons behind major packet drops.

- Collision rate defines the total number of packets collide per second. Packets delivery ratio will be high if collision rate is lesser which helps to increase the performance.

- Throughput rate parameter defines the average number of successful reached incoming and outgoing packets on a particular network.

Throughput $(\mathrm{KB} / \mathrm{sec})=\underline{\Sigma}$ (Number of incoming and outgoing packets at destination) $*$ Packet size $\Sigma$ Simulation Time

- Broadcasting rate parameter defines the broadcasting of incoming and outgoing packets on a specific network interface. All the packets broadcast over a network comes in broadcasting rate category.

\section{B. Simulation Scenario}

A scenario is created here with variable transmission power and different carrier sense power threshold conditions under mobile IP interface. In simulation two ray ground propagation models can be used which considers both the ground reflection path and direct path. Two ray ground gives more accurate prediction for long distance than free space model. Different RSU have different values of provider service identifier. All RSU have variable services and when vehicles comes in range of RSU then it takes only 1 second to register to that particular RSU. Dynamic path movement is selected to move vehicles to any direction at any time. Following commands can be added in application tab of mobile node $(\mathrm{MN})$ and $\mathrm{PC}$ to generate traffic.

Table 1. Passing Command between MN (Mobile Node) and PC

\begin{tabular}{|c|c|}
\hline Identification & Command \\
\hline $\begin{array}{c}\text { Traffic generation command at } \\
\text { sender side }\end{array}$ & $\begin{array}{c}\text { Stg }-\mathrm{i} \text { stg.conf 1.0.7.1-p } \\
2007\end{array}$ \\
\hline $\begin{array}{c}\text { Traffic generation command at } \\
\text { receiver side }\end{array}$ & Rtg- $\mathrm{u}-\mathrm{w} \log 1-\mathrm{p} 2007$ \\
\hline Moving vehicle command & Car Agent \\
\hline
\end{tabular}

Stg (Send traffic grapher) command is used to send traffic to both TCP and UDP nodes, it consists of IP address of receiver and it simulated the VoIP traffic where as Rtg (Receiver traffic grapher) command receives the traffic. Specifications of node information of scenario are listed in the table below.

Table 2. Node Information of the scenario

\begin{tabular}{|c|c|c|}
\hline $\begin{array}{c}\text { Node } \\
\text { ID }\end{array}$ & $\begin{array}{c}\text { Node } \\
\text { Identification }\end{array}$ & Node Description \\
\hline $\begin{array}{c}\text { Node } \\
12\end{array}$ & $\begin{array}{c}\text { OBU(For } \\
\text { moving nodes) }\end{array}$ & $\begin{array}{c}\text { Car Agent moving command is } \\
\text { used in application phase of IEEE } \\
802.11 \text { p OBU to generate traffic } \\
\text { and all nodes generate traffic at } \\
\text { receiver using rtg command. }\end{array}$ \\
$\begin{array}{c}\text { Node } \\
6,7,8,9\end{array}$ & $\begin{array}{c}\text { RSU (Road side } \\
\text { units) }\end{array}$ & $\begin{array}{c}\text { service information table is set with } \\
1 \text { second interval and home agent } \\
\text { and foreign agent is set in mobile } \\
\text { IP section. }\end{array}$ \\
\hline $\begin{array}{c}\text { Node } \\
10\end{array}$ & Router & $\begin{array}{c}\text { All RSU's are connected with } \\
\text { router. }\end{array}$ \\
\hline $\begin{array}{c}\text { Node } \\
11\end{array}$ & PC & $\begin{array}{c}\text { It generates traffic at sender side by } \\
\text { using stg command. }\end{array}$ \\
\hline
\end{tabular}


Various simulation parameters used in physical layer and channel model are deployed according to the channel requirement as shown in table below.

Table 3. Simulation parameters

\begin{tabular}{|c|c|}
\hline Parameters & Values \\
\hline Frequency (MHz) & 5860 \\
\hline Fading Variance & 10.0 \\
\hline Fading & Rayleigh \\
\hline Path loss model & Two Ray Ground \\
\hline System Loss & 1.0 \\
\hline Transmission Power (dbm) & $28.8,17.0$ \\
\hline Threshold (dbm) & $-80,-73$ \\
\hline Antenna Gain (dbi) & 1.0 \\
\hline MAC protocol & IEEE $802.11 \mathrm{p}$ \\
\hline Traffic tool & Stg, Rtg \\
\hline Mobility Model & Manhatten Grid \\
\hline Speed (Kmph) & 100 \\
\hline Simulation Time (sec) & 400 \\
\hline Data Rates (Mbps) & 8,16 \\
\hline Communication & Full Duplex \\
\hline Bit Error Rate & 0.01 \\
\hline
\end{tabular}

Using aforementioned simulation parameters simulations are performed using 'run' interface available in NCTUns 6.0 toolbar workplace.

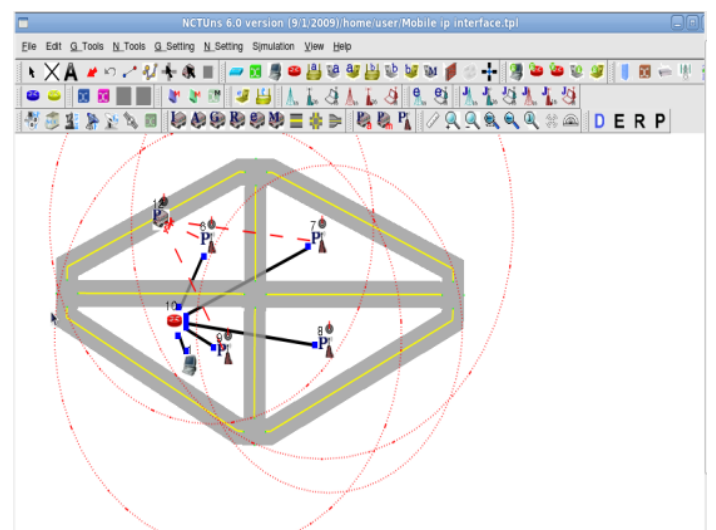

Fig.1. Mobile IP Scenario with OBU at home network.

\section{RESULT AND DISCUSSION}

This section outlines the result obtained from the evaluation of QoS parameters in the mobile IP interface. Subsection A shows the effect of changing carrier sense power threshold taking 28.8 power in mobile ip environment while subsection B shows light on effect of changing threshold taking 17.0 power in mobile IP realistic environment. In both these subsections Rayleigh fading is used to simulate realistic environment.

\section{A. Scenario 1: $Q o S$ at $R S U=28.8$}

The performance is examined in mobile IP interface taking threshold values -80.0 and -73.0 with 28.8 transmission power. Only values up to 40 seconds are taken in figure, yet table IV describes various average values up to 400 simulation time. Fig 2 shows Packet drop ratio (PDR) of threshold for power of value 28.8. During simulation two values of threshold -80.0 and 73.0 are taken. It has been examined that for threshold value -73 packet drops are slight higher as compared to other. As lower the packet drops higher will be the packet delivery ratio, here due to less threshold value in case of 73 the packets are dropping at fast rate. Fig 3 shows the collision rate of both threshold values in Rayleigh fading environment for 28.8 power values. As higher the collision rate more packets will drop and this affects the throughput. It has been examined that for -73 threshold value collision rate is slightly lower than for -80.0 . For 80.0 threshold value the collision rate has $3.15 \%$ average value slightly higher than $3.04 \%$. Fig 4 shows the throughput rate of incoming and outgoing packets for both threshold values. This figure examined that for threshold -73 the average value is $111.01 \%$ higher than $108.76 \%$ of -80 thresholds. Higher the throughput rate more will be the productivity. Fig 5 shows broadcast rate of incoming and outgoing packets of both threshold values for power 28.8. It has been examined that broadcast rate is higher for -80 threshold value by an average value of $4.63 \%$. It is concluded that for power 28.8 the threshold value i.e -73 has higher throughput rate and lesser collision rate yet there is slight downfall in packet drop.

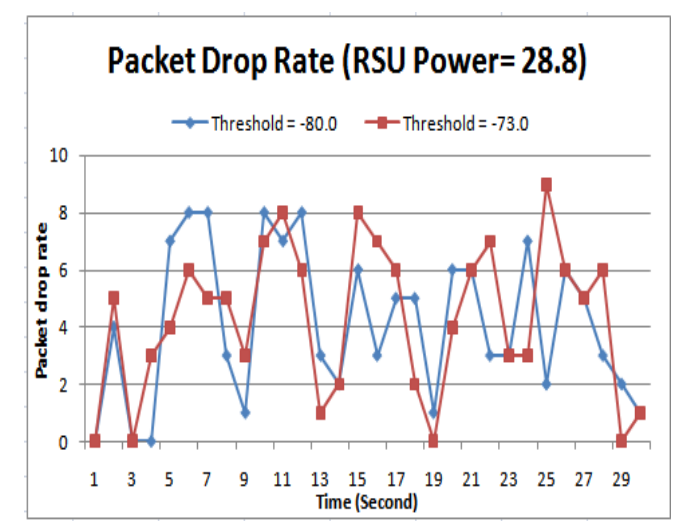

Fig.2. PDR ratio for power 28.8 in mobile IP environment for both threshold values

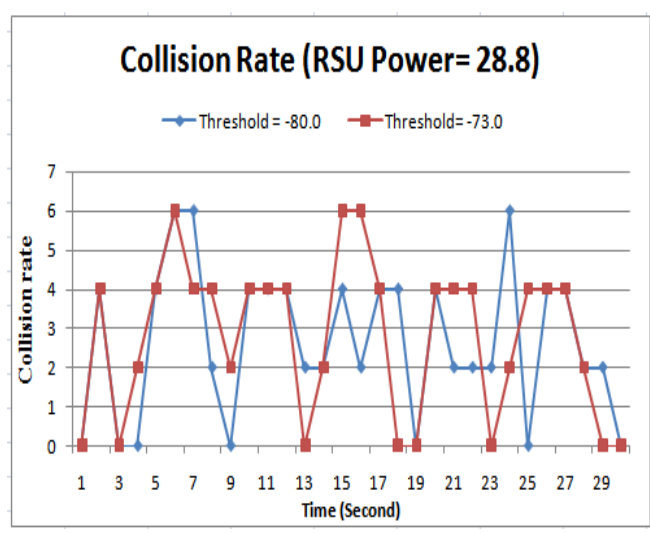

Fig.3. Collision ratefor power 28.8 in mobile IP environment for both threshold values 


\section{Throughput Rate (RSU Power= 28.8)}

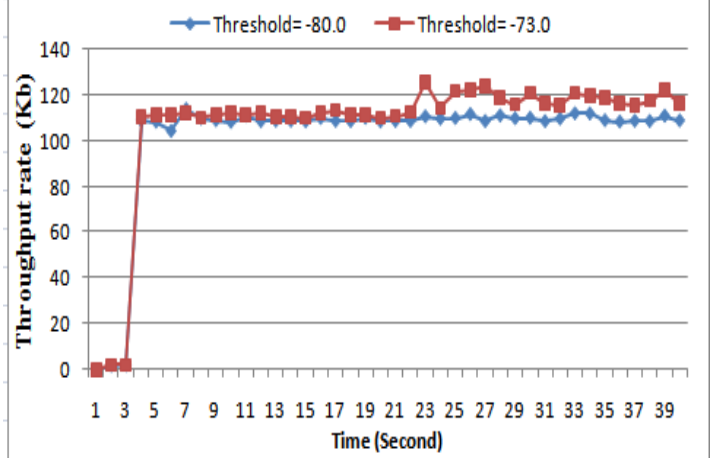

Fig.4. Throughput rate of incoming and out going packets for power 28.8 in mobile IP environment for both threshold values

\section{Broadcast Rate (RSU Power= 28.8)}

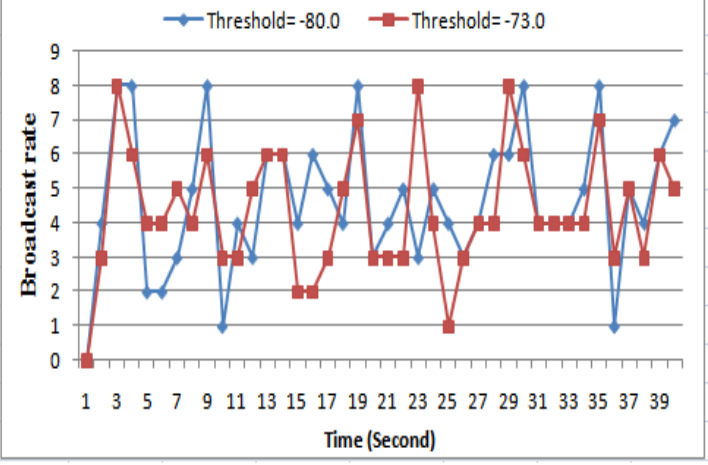

Fig.5. Broadcast rate of incoming and out going packets for power 28.8 in mobile IP environment for both threshold values

\section{B. Scenario 2: $Q o S$ at $R S U=17.0$}

The performance of VANET is analyzed in mobile IP environment for power 17.0 with variable carrier sense power threshold value while keeping speed constant at $100 \mathrm{kmph}$. Fig 6 shows packet drop ratio of both threshold values -80.0 and -73.0 for power 17.0 . It has been examined that for -73 thresholds value has $1.9 \%$ average value slightly higher than $0.43 \%$ average value of -80 thresholds. Fig 7 shows collision rate of both threshold values for power 17.0 and it is examined that -80 threshold values has slightly higher collision rate as compared to other threshold value. As greater the collision rate more the packet delivery ratio is effected and lesser will be the throughput. Fig 8 shows light on throughput rate of inco ming and outgoing packets of both threshold values for power 17.0 and examined that throughput rate for -73 value of threshold has average value of $128.9 \%$ very much greater than other. Higher throughput rate depicts greater delivery ratio and lesser packet drop ratio. Fig 9 show the broadcast rate of incoming and outgoing packets and examined that broadcast rate is higher for -80 threshold value by $3.0 \%$ average value. It is concluded that even if we decrease power to 17.0 the threshold value for -73 has higher throughput rate and lesser packet drop and collision rate. Table IV shows light on various average values of both thresholds at different power rates.

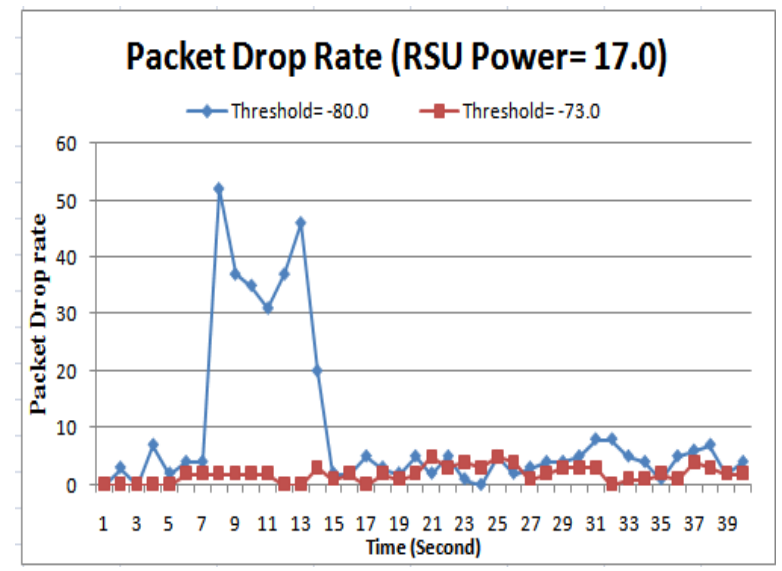

Fig.6. PDR ratio for power 17.0 in mobile IP environment for both threshold values.

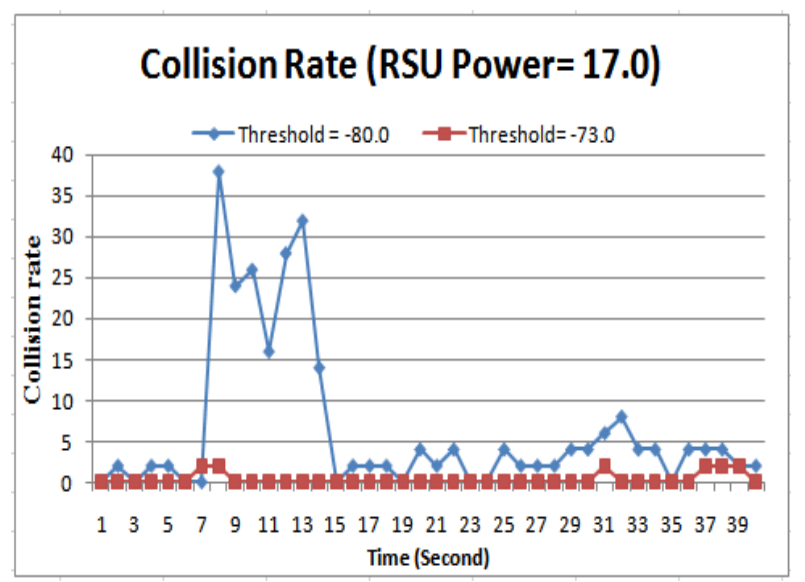

Fig.7. Collision ratefor power 28.8 in mobile IP environment for both threshold values

\section{Throughput Rate (RSU Power= 17.0)}

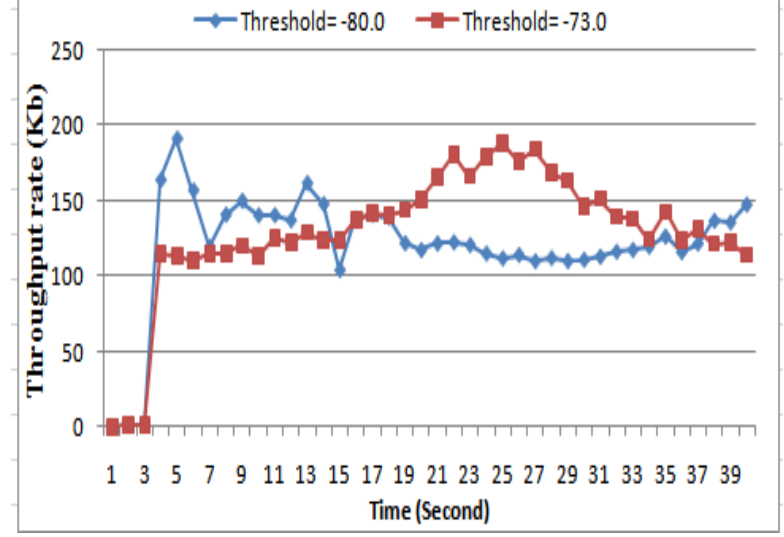

Fig.8. Throughput rate of incoming and outgoing packets for power 28.8 in mobile IP environment for both threshold values 


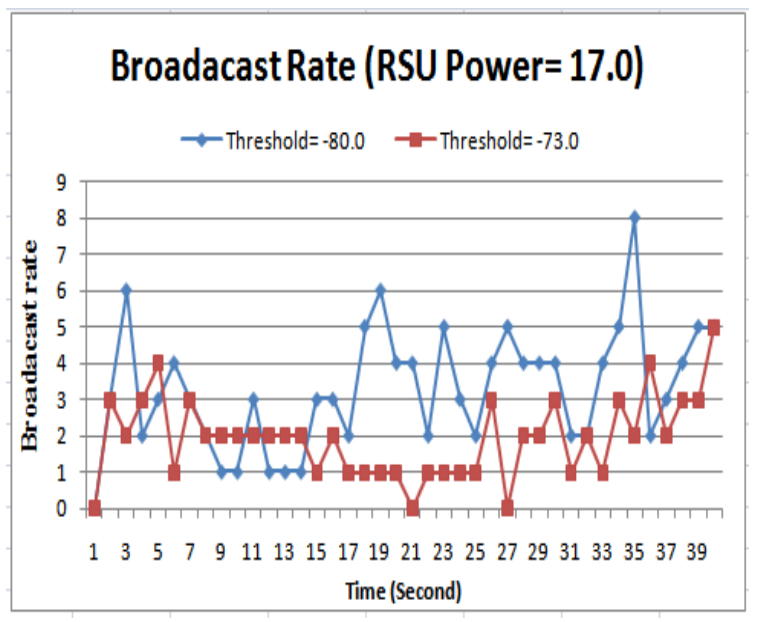

Fig.9. Broadcast rate of incoming and out going packets for power 28.8 in mobile IP environment for both threshold values

Table 4. Simulation results of various threshold values in mobile IP environment.

\begin{tabular}{|c|c|c|c|c|}
\hline \multicolumn{4}{|c|}{ Different average values in mobile IP environment } \\
\cline { 2 - 5 } & $\begin{array}{c}\text { Threshold } \\
\mathbf{- 8 0 . 0}\end{array}$ & $\begin{array}{c}\text { Threshold } \\
\mathbf{- 7 3 . 0}\end{array}$ & $\begin{array}{c}\text { Threshold } \\
\mathbf{- 8 0 . 0}\end{array}$ & $\begin{array}{c}\text { Threshold } \\
\mathbf{- 7 3 . 0}\end{array}$ \\
\hline $\begin{array}{c}\text { Packet } \\
\text { drop } \\
\text { ratio }\end{array}$ & 4.43 & 4.49 & 5.08 & 3.53 \\
\hline $\begin{array}{c}\text { Collision } \\
\text { rate }\end{array}$ & 3.15 & 3.04 & 2.91 & 1.93 \\
\hline $\begin{array}{c}\text { Through } \\
\text { put rate } \\
\text { (Kbps) }\end{array}$ & 108.76 & 111.01 & 126.45 & 127.5 \\
\hline $\begin{array}{c}\text { Broadcas } \\
\text { t rate }\end{array}$ & 4.63 & 4.53 & 3.65 & 2.64 \\
\hline
\end{tabular}

\section{CONCLUSIONS}

The main accent of this paper is to analyze the performance of QoS parameters for VANET in mobile IP environment using IEEE 802.11p MAC standard using different threshold and transmission power values. Using 28.8 transmission power the threshold value of -73 has greater throughput rate which helps to increase productivity and packet delivery ratio, also the collision rate is lower in case of -73 threshold. Yet there is slight downfall in packet drop. Whereas simulation results of 17.0 trans mission power has also higher throughput rate of $127.5 \%$ as compared to -80 threshold values. In this case also the packet drops and collision rate is greater for -80 thresholds. This concludes that even if we decrease power there are vibrantly good results for lesser threshold values. All the simulation results are incorporated using 400 simulation time and average values are calculated. These simulation results shows the acceptable improvement of QoS parameters in terms of throughput, packet drop and collision rate. VANET is a vast field and attracted much attention from research community, yet some issues need to be resolved like delay and improved path connectivity.

\section{ACKNOWLEDGMENT}

The authors wish to thank our friends, parents and faculty of GNDU RC Jalandhar for their valuable guidance and support.

\section{REFERENCES}

[1] Mahmood Fathy, Saeed Gholamalitabar Firouzjaee and Kaamran Raahemifar, "Improving QoS in VANET Using MPLS" in 7th International Symposium on Intelligent Systems Techniques for Ad hoc and Wireless Sensor Networks (IST-AWSN 2012).

[2] M. Samad, S.H.Herman, "Quality of Service for Mobile IP Services in Wireless Network", Asia Pacific Conference on Applied Electromagnetic, Johor, Malay sia, 2005.

[3] Sasan Adibi,Shervin Erfani, "Mobile Ad-hoc Networks With QoS and RSVP Provisioning", CCECE 2005,May 14 Saskatoon, Canada.

[4] Saeed GholamalitabarFirouzjaee, Mahmood Fathy and Kaamran Raahemifar, "Utilizing Mobile IP, MPLS to Improve QoS in VANET" International Conference on. Advances in Signal Processing and Communication (SPC2012) Amsterdam, Netherlands, Jun 8, 2012 pp- 122-125.

[5] Mr. Bhavin Gurjar "Detection of Failure Node and Safety to Vehicle using V2V Communication", National Conference on Recent Trends in Engineering \& Technology on $13^{\text {th }}$ may 2011.

[6] Cespedes. S, Taha. S. Xuemin Shen "A MultihopAuthenticated Proxy Mobile IP Scheme for Asymmetric VANET", Vehicular Technology, IEEE Transactions on 15 March 2013 Volume 62, Issue-7.

[7] A. Al Shidhani and V. C. M. Leung "Secure and efficient multi-hop mobile IP Registration scheme for MANETInternet integrated architecture", Proc. IEEE Wireless Communication Networking Conf, pp.1 -62010.

[8] Y.-C. Tseng, C.C. Shen, and W.T. Chen, "Integrating Mobile IP with Ad Hoc Networks," IEEE Computer, May 2003.

[9] M. Samad, S.H.Herman "Quality of Service for Mobile IP Services in Wireless Network", Asia Pacific Conference on Applied Electromagnetic, Johor, Malay sia, 2005.

[10] Prof. Shie-Yuan Wang,Chih-Liang Chou, Chih-Che Lin, and Chih-Hua Huang "The Protocol Developer Manual for the NCTUns 6.0 Network Simulator and Emulator", National Chiao Tung University, Taiwan.

[11] Marc Bechler, Lars Wolf, "Mobility Management for Vehicular Ad Hoc Networks" Vehicular Technologv Conference, 2005 VTC 2005-Spring. 2005 IEEE 61st Volume: 4, DOI: 10.1109 /VETECS 2005. 1543744 Publication Year 2005, Page(s): 2294 - 2298 Vol. 4.

[12] C. Sommer and F. Dressler "Using the right two-ray model, A measurement based evaluation of PHY models in VANETs", Proc. ACM MobiCom, pp.1 -3 2011.

[13] Muhammad Nawaz Khan, Ishtiaq Wahid, Gulzar Mehmood "A Global Mobility Management Scheme for Reducing Overall Handover Latency in IP based VANETs" International Journal of Ad hoc, Sensor \& Ubiquitous Computing (IJASUC) Vol.3, No.1, February 2012. 


\section{Authors' Profiles}

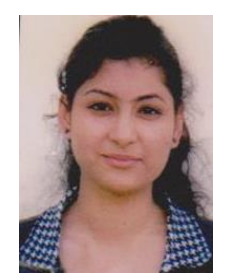

Richa Sharma received her B.Tech degree in Computer Science and Engineering from Punjab College of Engineering \& Technlogy, Punjab, India in 2012, pursing her M.Tech degree in Computer Science and Engineering with specialization in Vehicular Ad- Hoc Network from Guru Nanak Dev University, Amritsar, India. Her research interests include wireless communication and vehicular ad-hoc networks.

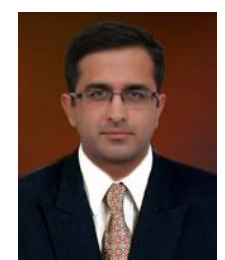

Dr Jyoteesh Malhotra, B.Engg.(with Distinction), M.Tech.(Gold Medalist), PhD (ECE), Head of CSE and ECE department GNDU RC Jalandhar, INDIA, Area of Specialization is Wireless / Optical Comm. Systems and Networks.

How to cite this paper: Richa Sharma, Jy oteesh Malhotra, "On Achievable QoS Bounds for Mobile IP through Rayleigh Channel in VANET", International Journal of Information Technology and Computer Science (IJITCS), Vol.8, No.8, pp.87-92, 2016. DOI: $10.5815 /$ ijitcs.2016.08.10 